\title{
Harmonic response from microscopic antibubbles
}

Michiel POSTEMA ${ }^{1,2,3, *}$, Anthony NOVELL ${ }^{1}$, Charles SENNOGA ${ }^{1}$, Albert T. POORTINGA ${ }^{4}$, Ayache BOUAKAZ ${ }^{1}$ 


\title{
Harmonic response from microscopic antibubbles
}

\author{
Michiel POSTEMA ${ }^{1,2,3, *}$, Anthony NOVELL ${ }^{1}$, Charles SENNOGA ${ }^{1}$, \\ Albert T. POORTINGA ${ }^{4}$, Ayache BOUAKAZ ${ }^{1}$ \\ ${ }^{1}$ Inserm Research Unit U1253: Imaging and Brain, UFR Médecine, \\ 10 Boulevard Tonnellé, 37032, Tours, France \\ ${ }^{2}$ LE STUDIUM Loire Valley Institute for Advanced Studies, 1 Rue Dupanloup, 45000, \\ Orléans, France \\ ${ }^{3}$ School of Electrical and Information Engineering, University of the Witwatersrand, \\ Johannesburg, 1 Jan Smuts Laan, Braamfontein 2050, South Africa \\ ${ }^{4}$ Department of Mechanical Engineering, Eindhoven University of Technology, 5612 AZ \\ Eindhoven, The Netherlands
}

\begin{abstract}
An antibubble is a gas bubble containing a liquid droplet core. Both the droplet and the gas bubble are typically surrounded by stabilising shells. Owing to electrostatic forces exerted by these shells, core droplets of micrometer diameter do not readily coalesce with the surrounding liquid medium. Owing to the incompressibility of the liquid droplet core, antibubbles will oscillate asymmetrically, i.e., the radial excursion amplitude of the surface is greater during expansion than during contraction, when subjected to diagnostic ultrasound. Consequently, the harmonic content of the ultrasound signal radiated from antibubbles must be higher that that from identical bubbles without a liquid core. Whether the harmonic signal component generated by physical antibubbles is higher than the harmonic component of identical bubbles without a core has been studied here. We subjected prefabricated antibubbles and identical bubbles without core droplets to $1-\mathrm{MHz}$ ultrasound and to a commercial ultrasound system, and recorded the spectra with a broadband transducer oriented perpendicularly to the transmitter. Normalised by the acoustic response from the medium, the antibubble signal shows stronger higher harmonics than the reference signal, and negligible fundamental response. In conclusion, antibubbles are suitable candidates for harmonic imaging. The generation of higher harmonics without fundamental has been attributed to asymmetric antibubble expansion.
\end{abstract}

Key words: Microbubbles, ultrasound contrast agent, imaging, ultrasonics. 


\section{Introduction}

An antibubble is a gaseous bubble comprising a trapped liquid droplet at its core ( $c f$. Figure 1). Both the gas-liquid interfaces of the droplet and the bubble are stabilised by jammed packings of hard particles [1]. Owing to electrostatic forces exerted by these stabilised shells, core droplets of micrometer diameter are prevented from readily coalescing with the surrounding liquid medium. Following their appearance in scientific literature [2], antibubbles have been under investigation as vehicles for ultrasound-guided drug delivery [3] and magnetic delivery [4]. It has also been hypothesised that, because of the structural arrangement, antibubbles can be suitable agents for harmonic imaging [5].

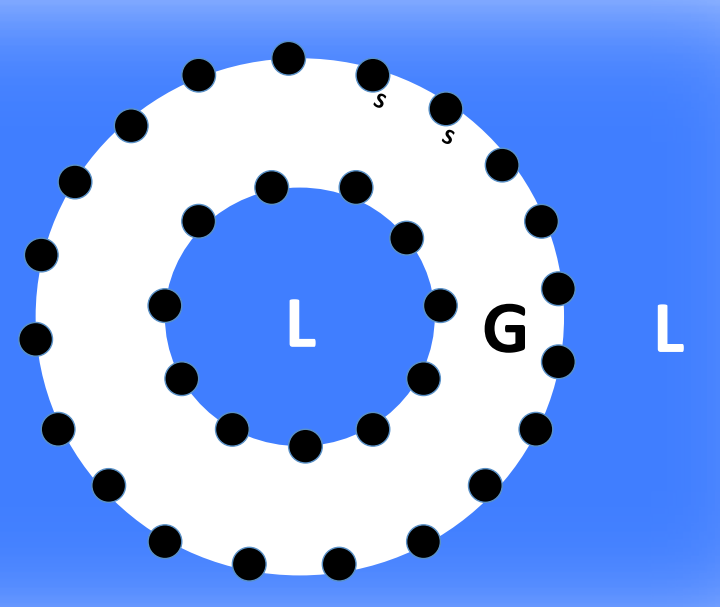

Figure 1: Schematic representation of a liquid medium with an antibubble, consisting of a liquid (L) droplet surrounded by a gas $(\mathrm{G})$ layer. The inner and outer shells contains solid (S) nanoparticles. 
Owing to the incompressibility of the liquid droplet core, antibubbles with a droplet core greater than a critical size must oscillate asymmetrically, i.e., the radial excursion amplitude of the outer antibubble surface is greater during expansion than during contraction, when subjected to diagnostic ultrasound [5]. Consequently, the harmonic content of the ultrasound signal radiated from antibubbles must be higher than that from identical bubbles without a liquid core.

In a separate simulation study, we numerically determined that the critical size at which the harmonics can be discriminated is at droplet diameters around $20 \%$ of the outside diameters [6]. Below this ratio, the harmonic component was too small to be detected. Examples of numerical simulations of the acoustic response from bubbles and antibubbles without an encapsulating shell have also been presented in [7], indicating a significant higher harmonic content of antibubbles compared to conventional bubbles.

The question of whether the harmonic signal component generated by physical antibubbles is higher than the harmonic component of identical bubbles without a core is the focus of this paper.

The scattering response of antibubbles and reference bubbles to $1-\mathrm{MHz}$ ultrasound was measured and compared. In addition, the feasibility of creating a harmonic image with a commercial ultrasound system was tested. 


\section{Materials and methods}

We prepared three media for this evaluation.

The first compound studied was an emulsion with antibubbles having liquid cores of $20-\mu \mathrm{m}$ diameter, produced as previously published [8]. The antibubbles were stabilised using Aerosil@ R 972 hydrophobized silica particles (Evonik Industries AG, Essen, Germany). The silica particles, with diameters below $30 \mathrm{~nm}$, were observed to form a single elastic layer on the antibubble surface on which the individual particles could not be discriminated. The aqueous cores were replaced by 2 vol\% of hydrophobically modified Zano 10 Plus zinc oxide nanoparticles (Umicore, Brussel, Belgium) with mean diameters of $30 \mathrm{~nm}$. These were observed to form a tightly packed layered structure on the antibubble core surface.

The second compound studied consisted of an emulsion with bubbles, identical to the antibubbles studied, but without liquid cores. These bubbles are referred to as reference bubbles in this paper. Reference bubbles were produced in the same way as the antibubbles, just without adding core material.

In addition, a medium consisting of just saline was similarly studied. The experiments with saline were performed as null experiments.

From the two compounds evaluated, $14 \mathrm{mg}$ of freeze-dried material was diluted in $1 \mathrm{ml}$ of Otec ${ }^{\mathrm{R}}$ 0,9\% saline (LABORATOIRE AGUETTANT, Lyon, France) and shaken for $60 \mathrm{~s}$ in a CapMix ${ }^{\mathrm{TM}}$ (3M ESPE, Seefeld, Germany). From these emulsions, $20 \mu \mathrm{l}$ was pipetted into a Fisherbrand ${ }^{\circledR}$ FB55143 macro cuvette (Fisher Scientific SAS, Illkirch, France) containing $3 \mathrm{ml}$ saline. The cuvette was then placed centrally in a container filled with degassed water.

The experimental setup used was presented in a previous study [9]. Briefly, an unfocussed transmitting single-element transducer was mounted to one side and a wide-band receiving single-element transducer was mounted perpendicularly to the transmitting transducer. A metal frame was attached to the container so that the media under investigation could be positioned precisely. In each experiment, a pulse from a 5077PR square wave pulser/receiver (Olympus Corporation, Shinjuku, Tokyo, Japan) was triggering a 33220A arbitrary waveform generator (Agilent Technologies, Santa Clara, CA, USA), which generated 20 cycles of a 1-MHz, $100 \mathrm{mV}$ peak-to-peak signal. The signal was attenuated with a 75-A-MFN-03 75-W attenuator (Foshan Yixun Co Ltd, Longjiang, PR China) and subsequently amplified by an AAP-500- 
0.2-6-D 500-W power amplifier (ADECE, Veigne, France). The signal was transmitted with an unfocussed custom 1-MHz transmitting single-element transducer (SOFRANEL, Sartrouville, France) with a 13-mm diameter. The corresponding peak-to-peak pressure of the ultrasound signal was calibrated at $220 \mathrm{kPa}$. A custom 2.25-MHz wide-band receiving single-element transducer (SOFRANEL, Sartrouville, France) with a 51-mm diameter and focussed at $55 \mathrm{~mm}$ was mounted perpendicularly to the transmitting transducer. The received signal was recorded using a TDS 3044B digital oscilloscope (Tektronix, Beaverton, OR, USA).

Thirty identical experiments were performed for each of the three media studied, i.e., the antibubbles, the reference bubbles, and the saline.

Using the Fast Fourier Transform, frequency spectra of the recorded signals were computed in MatlaB ${ }^{\circledR}$ (The MathWorks, Inc., Natick, MA, USA). For each medium studied, the response of the 30 experiments was averaged. The resulting curves were normalised by the response of saline after which spectral noise was removed with a five-point running smoother.

In addition, the antibubbles and reference bubbles were inserted in 12cm long I3659 transparent straws (Farnell element14, Leeds, United Kingdom) with a $6.40-\mathrm{mm}$ diameter, which were clamped on a metal frame in a polystyrene tank filled with degassed water. At a distance of $1 \mathrm{~cm}$ above the straw under investigation, a SuperLinear ${ }^{\mathrm{TM}}$ 15-4 ultrasound probe (Supersonic Image, Aix-en-Provence, France) was fixed, connected to an Aixplorer ${ }^{\circledR}$ ultrasound imaging system (Supersonic Image, Aix-en-Provence, France). The imaging system operated between frequencies of $4 \mathrm{MHz}$ and $15 \mathrm{MHz}$. 


\section{Results and Discussion}

The results have been summarised in Figure 2. The reference bubbles show a wide-band fundamental as well as higher order harmonics. However, the fundamental mode is absent from the antibubble response whilst higher harmonics up to the fourth order harmonic are evident.

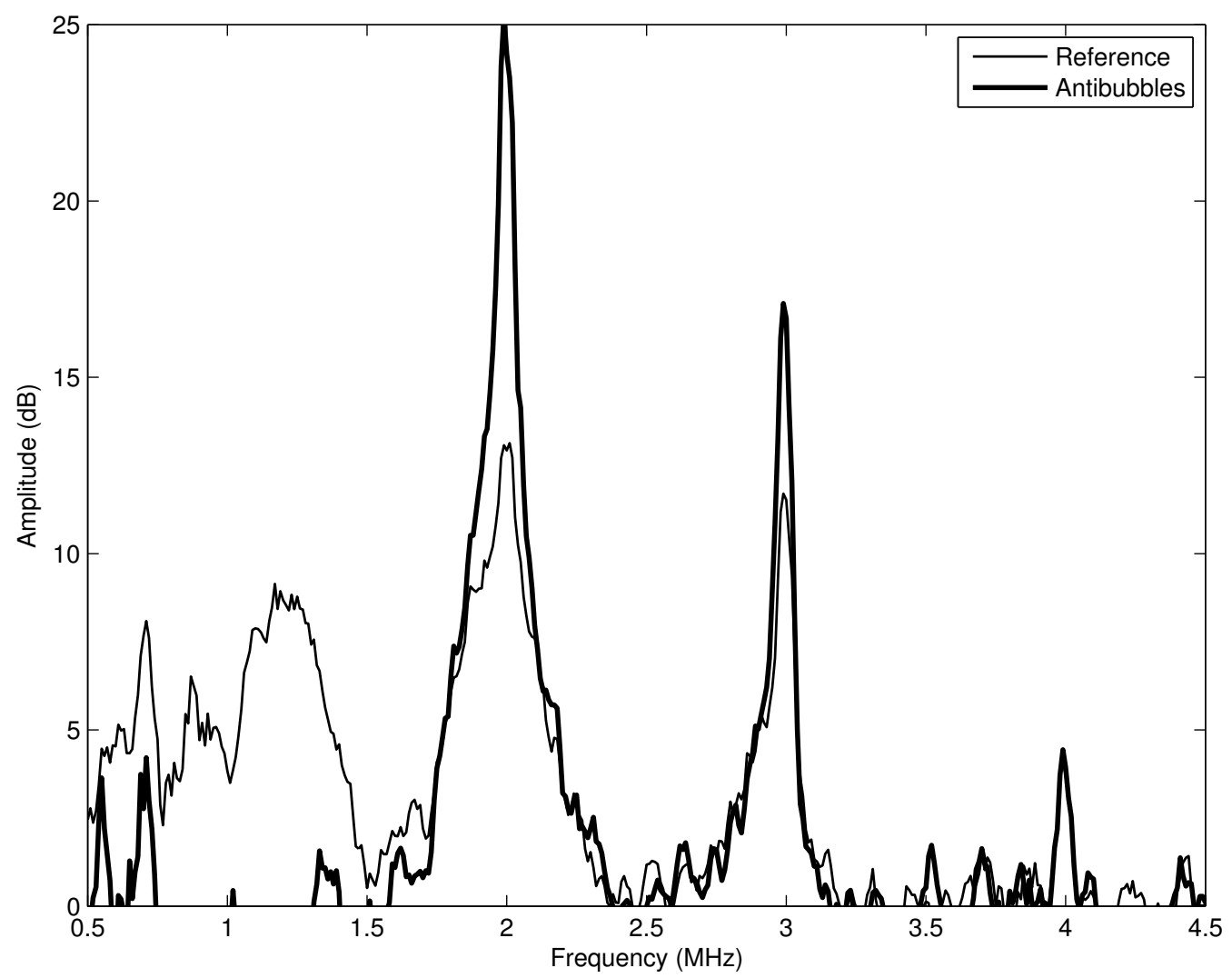

Figure 2: Fourier spectrum of the antibubble response (bold) and reference bubbles (thin), normalised by the response of the medium alone: received amplitude in $\mathrm{dB}$ as a function of frequency in $\mathrm{MHz}$.

These results indicate the potential of antibubbles in harmonic imaging. Furthermore, the absence of wide-band noise indicates that the antibubbles and reference bubbles have remained intact during the experiments.

It must be noted that the responses have been normalised in the frequency domain by the narrowband response from the medium alone. Consquently, the anticipated peak around $1 \mathrm{MHz}$ is absent from the response of the reference 
bubbles, resulting in a lower fundamental than harmonic response in the spectrum.

As the antibubbles are of identical construct as the reference bubbles except for the presence of a core, the higher harmonics from the antibubbles can be attributed to the asymmetry between expansion and contraction predicted from theory.

The frequencies used in these first experiments were chosen with the knowledge of the average size of the antibubbles and reference bubbles. However, $1 \mathrm{MHz}$ would be below typical frequencies used in medical imaging. Clearly, with outer diameters of $20 \mu \mathrm{m}$, these antibubbles are too large to be used in a clinical setting. A next generation of antibubbles may be developed to overcome this shortcoming.

Figure 3 demonstrates brightness-mode (B-mode) images of reference bubbles and antibubbles inside an optically transparent tube. The acoustic amplitude corresponded to a mechanical index of 0.1 . Reflections from the top of the tube can be seen to the right-hand side of the frames, at depths of approximately $1 \mathrm{~cm}$. In the image of the reference bubbles (frame a), only scatterers close to the surface are visible, while In the image of the antibubbles (frame b), scattering from the medium on the left-hand side inside the tube is visible. This scattering is attributed to the presence of the antibubbles. It is noted that at higher acoustic amplitudes, scattering from both bubbly media has been observed.

It will be interesting, albeit beyond the scope of the study, to compare differences in signals of fragmenting or coalescing antibubbles and reference bubbles. 

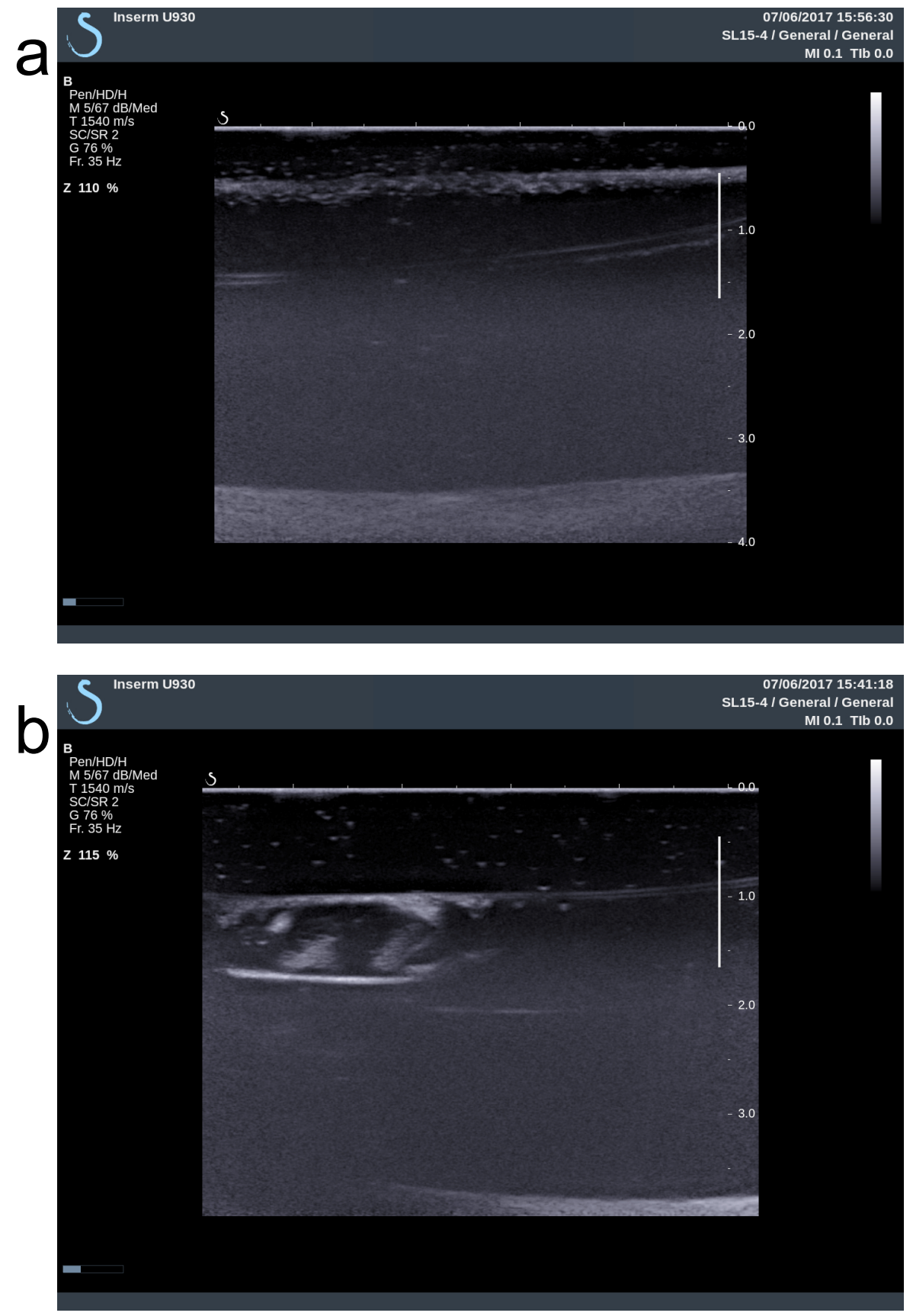

Figure 3: B-mode scans in harmonic mode of reference bubbles (a) and antibubbles (b), at a set mechanical index of 0.1 . 


\section{Conclusion}

In conclusion, antibubbles are suitable candidates for harmonic imaging. The generation of higher harmonics without fundamental has been attributed to asymmetric antibubble expansion.

With a commercial ultrasound imaging system, antibubbles have been visualised in an in-vitro setup, at mechanical index as low as 0.1.

\section{Acknowledgements}

M.P. has received funding from European Union's Horizon 2020 research and innovation programme under Marie Skłodowska-Curie grant agreement No 665790 .

${ }^{*}$ Correspondence and requests for materials should be addressed to M.P. (email: michiel.postema@wits.ac.za).

\section{References}

[1] Poortinga AT. Long-lived antibubbles: stable antibubbles through Pickering stabilization. Langmuir 2011;27:2138-41.

[2] Dorbolo S, Caps H, Vandewalle N. Fluid instabilities in the birth and death of antibubbles. New J Phys 2003;5:161.1-9.

[3] Postema M, ten Cate FJ, Schmitz G, de Jong N, van Wamel A. Generation of a droplet inside a microbubble with the aid of an ultrasound contrast agent: first result. Lett Drug Des Discov 2007;4:74-7.

[4] Silpe JE, McGrail DW. Magnetic antibubbles: formation and control of magnetic macroemulsions for fluid transport applications. J Appl Phys 2013;113:17B304.

[5] Johansen K, Postema M. Lagrangian formalism for computing oscillations of spherically symmetric encapsulated acoustic antibubbles. Hydroacoustics 2016;19:197-208.

[6] Johansen K, Kotopoulis S, Poortinga AT, Postema M. Nonlinear echoes from encapsulated antibubbles. Phys Proc 2015;70:1079-82.

[7] Kotopoulis S, Johansen K, Gilja OH, Poortinga AT, Postema M. Acoustically active antibubbles. Acta Phys Pol A 2015;127:99-102. 
[8] Poortinga AT. Micron-sized antibubbles with tunable stability. Colloid Surface Physicochem Eng Aspect 2013;419:15-20.

[9] Novell A, Escoffre JM, Bouakaz A. Second harmonic and subharmonic for non-linear wideband contrast imaging using a capacitive micromachined ultrasonic transducer array. Ultrasound Med Biol 2013;39:1500-12. 